\title{
BULLYING Y LA VIOLENCIA CONTRA LOS ADOLESCEN- TES EN LA FAMILIA Y SU IMPACTO EN LA AGRESIÓN EN LA ESCUELA: \\ Caso de estudio de las preparatorias técnicas en Nuevo León, Mexico
}

\author{
Claudia Elizabeth GONZALEZ AMAYA; Arun Kumar ACHARYA; \\ José María INFANTE BONFIGLIO \\ Universidad Autónoma de Nuevo León (México)
}

lizi.amaya@gmail.com, arun.kumar@uanl.mx, jose.infanteb@uanl.mx

BULLYING AND VIOLENCE AGAINST ADOLESCENTS IN THE FAMILY AND THEIR IMPACT ON AGGRESSION AT SCHOOL: Case study of technical high schools in Nuevo León, Mexico

Resumen: Diferentes tipos de violencia en la familia dan lugar a conductas agresivas entre los adolescentes y éstas suelen verse reflejadas en su comportamiento en las escuelas. El presente artículo investiga estos tipos de violencia y examina los factores familiares que originan que los adolescentes reaccionen con comportamientos violentos en su centro escolar. Para analizar este fenómeno se entrevistaron a 210 alumnas de tres preparatorias técnicas ubicadas en el Estado de Nuevo León, México en el período comprendido de Enero a Junio de 2016. Resultados señala que; la estructura familiar actualmente atraviesa por un importante periodo de crisis y cada vez son más los casos de familias las cuales se distinguen por ser disfuncionales e inseguras y esta situación atenta con destruir lo que en teoría debería ser un entorno familiar, sin embargo cabe señalar que la familia constituye un componente de vital importancia para las sociedades civilizadas. De hecho, podríamos asumir que en un breve periodo de tiempo hemos pasado de una tradicional familia unida, a una en la cual los integrantes son más independientes, desde temprana edad, y de esta manera caen en el consumo de alcohol y/o drogas afectando además su rendimiento académico.

Abstract: Different types of violence in the family lead to aggressive behavior among adolescents and these are often reflected in their behavior in schools. This article investigates these types of violence and examines the family factors that cause adolescents to react in a violent behavior in their school. To analyze this phenomenon, we interviewed 210 students from three technical high schools located in the State of Nuevo León, Mexico in the period from January to June 2016. Results indicates that; the family structure is currently going through an important period of crisis and each time there is increasing number of dysfunctional and insecure families, and this situation is intent on destroying what in theory should be a family environment. However it should be noted that the family constitutes a vital component for civilized societies. In fact, we could assume that in a short period of time we have moved from a traditional family unit, to one in which the members are more independent, from an early age, and thus fall in the consumption of alcohol and / or drugs affecting. In addition their academic performance.

Palabras clave: Familia. Bullying. Violencia escolar. Adolescentes. Nuevo León. México Family. Bullying. Violence in school. Adolescents. Nuevo Leon. Mexico 


\section{Introducción}

La Organización Mundial de la Salud (OMS) define la violencia como el uso deliberado de fuerza física o el poder, ya sea en grado de amenaza o efectivo, contra uno mismo, otra persona o un grupo o comunidad, que cause o tenga muchas probabilidades de causar lesiones, muerte, daños psicológicos, trastornos mortales, del desarrollo o privaciones (Amemiya, Oliveros y Barrientos, 2009 en Almeida y Gómez, 2005, Amaya et.al., 2016). Se ha establecido un cierto tipo de consenso internacional para definir la violencia, el cual incluye varios niveles claves como ataques, o intimidaciones físicas, verbales o psicológicas, que están destinadas a causar miedo, dolor o daño a la víctima, abuso de poder de una relación de poder asimétrica, desde el más fuerte hacia el más débil, ausencia de provocación por parte de la víctima y repetidos incidentes en los mismos niños o jóvenes a través de un prolongado y sostenido periodo de tiempo (Swain, 1998 en Almeida y Gómez, 2005).

Algunos autores (Varela, Ávila y Martínez, 2013 en Acevedo, 2012, Acharya, 2016) comentan que en la actualidad, la mayoría de los investigadores comparten la idea de que la violencia escolar es un tema complejo, el cual obedece a factores individuales, sociales, familiares y escolares, y se cree que es necesario adoptar una visión holística y ecológica del problema. Por lo tanto, es importante el análisis teniendo en cuenta dichos factores y estableciendo la diferencia entre hombres y mujeres. La conducta violenta de los adolescentes escolarizados se relaciona con los factores individuales (autoestima, soledad, satisfacción con la vida, distrés psicológico y empatía), familiares (cuma social familiar y comunicación con la madre y el padre), escolares (clima social en el aula, actitudes hacia la autoridad, violencia y victimización en la escuela) y comunitarios (integración, participación, sistemas informales y sistemas formales) y con el hecho de que existen grandes similitudes en el grupo de adolescentes más violentos en función del sexo. Considerando lo anterior, el presente artículo tiene como objetivo analizar cómo diferentes tipos de violencia en la familia generan actitudes agresivas entre las adolescentes, las cuales se ven manifestadas en los espacios públicos como las escuelas. Para el presente estudio se entrevistaron a 210 alumnas de tres preparatorias técnicas ubicadas en el Estado de Nuevo León en el período comprendido de Enero a Junio de 2016. Dicha investigación se realizó mediante un cuestionario semiestructurado el cual fue aplicado en la Escuela Industrial y Preparatoria Técnica Álvaro Obregón, la Preparatoria No. 20 de la Universidad Autónoma de Nuevo León (UANL) y el CONALEP 1 "Ing. José Antonio Padilla Segura”.

\section{El bullying, violencia escolar y su percepción social}

El término sajón bullying, según Acevedo (2012) empezaría a utilizarse en Suecia y en Noruega a partir de la introducción del concepto por Heinemann, utilizando la versión sueca de un libro del etiólogo Konrad Lorenz que se refería a un ataque colectivo por un grupo de animales sobre un animal de otra especie que constituye un enemigo natural, o a la acción de un grupo escolar contra un individuo desviado del grupo, analogía que nos ayuda a comparar esta situación con el diario de muchos niños y adolescentes que se ven obligados a sobrevivir en un contexto escolar violento en el cual la debilidad de uno, constituye la fortaleza del otro y es en esta situación en donde la violencia encuentra un terreno fértil para desarrollarse.

En relación a lo anterior, sucede que los medios de comunicación han retratado en sus desplegados periodísticos, noticieros y documentales el llamado clima de violencia, mediante ilustraciones de intimidación, corrupción, agresiones, secuestros, enfrentamientos y ajustes de cuentas por el crimen organizado; en las extorsiones y la presencia cada vez más crítica de la inseguridad pública, violencia que según Zizek (2008) es clasificada como vio- 
lencia subjetiva, que es aquella más aparatosa, en la cual el agresor tiene una postura bien establecida frente a su víctima. Lo anteriormente mencionada, son realidades que actualmente forman parte de la vida cotidiana de los habitantes en sus distintos contextos: ya sea en la esquina, en la colonia o barrio, en la escuela, en el trayecto a casa o centro de trabajo, o en cualquier escenario donde se genere cualquier tipo de interacción entre individuos siendo éstos testigos de manifestaciones de violencia las cuales atentan contra la armonía social.

Por otra parte, hablando de situaciones que afectan la armonía social definitivamente es preciso mencionar las conductas antisociales, las cuales se pueden manifestar en una amplia gama de acciones, como: agresiones, robos, vandalismo, piromanía, mentiras, ausentismo escolar y huidas de casa. En los niños y adolescentes, las conductas antisociales se pueden manifestar en agresiones, peleas con golpes e insultos, mentiras, consumo de drogas, no someterse a las reglas y expectativas sociales que son relevantes, atentando con ello en el entorno de las personas y las propiedades. En ocasiones la conducta antisocial se torna más gravosa en la medida en que el adolescente empieza a confrontar los sistemas de justicia penal, situación que se genera al pasar de una conducta antisocial a una conducta delictiva. La conducta antisocial incluye las acciones que realiza el sujeto, como pueden ser: disrupción, alteración del orden, en centros educativos -acciones de baja intensidad que interrumpen el ritmo de las clases-; los problemas de disciplina -conflictos entre profesor/alumno, jefe/ empleado, padre/hijo-; el maltrato entre iguales; el vandalismo; la violencia física; el acoso o violencia sexual; actos delictivos y la violencia perversa; así como el bullying, entre otros. Por otra parte se destaca la necesidad de avanzar en el estudio y la reflexión de cada una de las diferentes aristas del clima de la violencia, contribuyendo así a la generación de modelos preventivos que puedan contrarrestar las inercias y reproducciones sociales de perfiles altamente delictivos, que lo único que están produciendo, aparte de agudizar el clima de la violencia, es la saturación de los centros penitenciarios y la incorporación de los jóvenes al crimen organizado (Acevedo, 2012, Acharya y stevanato, 2005).

\section{Construcción social de la violencia y su impacto}

El Modelo Ecológico resulta ser útil de manera especial cuando se trata de llevar a cabo un análisis de la violencia ya que constituye un enfoque el cual explora la relación entre los factores individuales y contextuales del individuo considerando a la violencia como producto de muchos niveles de influencia sobre el comportamiento. Además el Modelo Ecológico destaca que los niveles de tipo individual pretenden identificar los factores biológicos y de la historia particular los cuales influyen en el comportamiento de una persona; el nivel de las relaciones sociales con los amigos, pareja y otros miembros de la familia, aumenta el riesgo de convertirse en víctimas o agresores; el nivel de la colectividad examina los contextos de la comunidad en los que se inscriben las relaciones sociales, tales como la escuela, el lugar de trabajo, el vecindario; además busca identificar las características de esos ámbitos que se asocian con ser víctimas o agresores y finalmente el nivel social examina los factores sociales que con mayor frecuencia determinan las altas tasas de violencia (Magenzo y Tchmino, 2010 en Acevedo, 2012).

En este mismo sentido, es importante destacar que la violencia es hoy un componente cotidiano en nuestras vidas, representa una manifestación que ocurre en todos los niveles sociales, económicos y culturales y, de manera particular, en las instituciones escolares. No podemos negar el hecho de que en ciertas ocasiones, y debido a ciertos intereses los cuales pueden ser de cualquier naturaleza, la violencia ha sido ocultada, negada y silenciada durante muchos años por educadores y autoridades; sin embargo, evitar y suprimir esos actos violentos no ha hecho más que empeorarlos, por lo cual el problema debe tomarse con firmeza en toda su magnitud (Oliveros, Figueroa, Mayorga, Cano y Barrientos, 2008 en Acevedo, 2012). 
En el marco de las observaciones anteriores (Arellano, 2007 en Acevedo, 2012) agrega que cuando se habla de violencia debe verse desde dos perspectivas íntimamente relacionadas, como lo son la violencia indirecta y la violencia directa. La primera se relaciona con las prácticas de indiferencia de los actos violentos o la no denuncia de los mismos, tiene un carácter impersonal y gran carga de apatía; la segunda reviste un carácter personal, cara a cara, y es donde se utiliza la fuerza física o la palabra, como medio para resolver frustraciones y diferencias, mostrándose de diversas maneras, como puede ser a nivel corporal, psicológico o moral, también se expresa contra objetos y ambiente. Barbeito y Caireta (2005 en Acevedo, 2012) enuncian que la violencia directa se puede asumir de igual manera como la actitud o el comportamiento que constituye una violación o una privación al ser humano de una cosa que le es esencial como persona, como puede ser la integridad física, psíquica o moral, sus derechos y libertades.

Por su parte, otros autores como Rodríguez y Palomero (2001 en Acevedo, 2011) coinciden en afirmar que la violencia se da de manera indirecta o estructural y, por ende, incorporada al sistema económico-social, manifestándose de manera concreta en injusticias sociales, como es el caso de la existencia de una diferencia en la distribución del ingreso, acompañada de marginalidad, analfabetismo, carencia de servicios médicos asistenciales, y dificultad de ascenso socioeconómico en altos porcentajes de la población. Es decir se podrían vincular los efectos de la violencia con el hecho de impedir el desarrollo de las potencialidades del ser humano, por lo tanto es preciso enfocar la violencia como la causa de la diferencia entre lo que las personas podrían ser, pero no son: entre lo actual y lo potencial, en cuanto a lo que se refiere a la realización de una vida mínimamente humana (Lederach, 1998 en Acevedo, 2012).

En relación a lo anterior, la violencia no es un concepto unívoco ni designa a un fenómeno singular; por el contrario, involucra la existencia de múltiples violencias con implicaciones a niveles individuales, familiares, institucionales y sociales, los cuales se articulan potenciando o moderando sus manifestaciones. Bringuiotti (2000 en Acevedo, 2012) señala en dicho sentido que, si bien hay formas históricas y culturales que caracterizan la violencia de nuestro tiempo, sus prácticas son producto de configuraciones particulares en los distintos escenarios. Por su parte Alegre (2004 en Acevedo, 2012) establece que actualmente en las instituciones educativas es donde se incrementa la violencia escolar la cual se entiende como cualquier situación de agresión física, verbal y emocional ejecutada por los alumnos, maestros y la administración y a su vez todo esto está relacionado con modalidades y canales según el grado de relación ya sea política, institucional, comunicacional y personal, y que juntos los anteriores elementos, propicien el clima de violencia dándose lugar a la presencia de la acción violenta (Luciano, Marín y Yuli, 2008 en Acevedo, 2012).

Por otro lado, es importante destacar, el hecho de que la mujer ejerce la violencia, en primera instancia en contra de sí misma ya que la relación más importante de todo individuo es la que desarrolla consigo mismo y según lo anterior, algunos expertos señalan que dicha relación no es muy bien manejada por las mujeres argumentando que éstas, son duras al calificarse, afectándose de diversas formas. De pronto algunas mujeres tienden a creer que si tuvieran suficientes logros, si fueran más esbeltas o mucho más bellas según su percepción, o tuvieran más dinero podrían modificar el propio diálogo interno y convertirlo en una declaración de fortaleza y riqueza espiritual (Ludwig y Marble 2004).

Resulta oportuno señalar que La National Science Foundation descubrió que la persona promedio llega a tener hasta sesenta mil pensamientos al día y que son, en su mayoría negativos y críticos. Es decir, es probable que de la estadística anteriormente mencionada, no todos los pensamientos sean realmente objetivos o reales, sin embargo, según los datos arrojados por la investigación la persona que tiene dichos pensamientos los considera como verdades, las cuales se repite una y otra vez hasta que termina por creerlas. Dicha batalla interna podría mermar la autoestima conduciendo a la persona a estados depresivos, insa- 
tisfacción, baja productividad y un alto grado de estrés que podría después somatizarse y convertirse en algún tipo de padecimiento físico. A continuación algunas estadísticas:

- El National Institute of Health reporta que 70 por ciento de las enfermedades están asociadas al estrés.

- La revista Time señala que en la actualidad, las mujeres son más poderosas pero menos felices que antes.

Por otra parte, en The Paradox of Declining Female Happiness, Betsy Stevenson y Justin Wolfers informan: "Considerando diversas mediciones objetivas, la vida de las mujeres en los Estados Unidos ha mejorado en los últimos 35 años; sin embargo, aquí mostramos que las medidas del bienestar subjetivo indican que la felicidad de las mujeres ha disminuido de manera absoluta y relativa en contraste con la de los hombres" (Ludwig y Marble, 2004).

En relación a lo anterior, Ahlers (2011) señala que la falta de amor y aprobación de las mujeres para sí mismas, impacta en su salud ya que diversas instituciones de salud mental reportan que en Estados Unidos, la cifra de mujeres con depresión duplica a la de los hombres; señalan que entre cinco y diez millones de mujeres (jóvenes y adultas), sufren trastornos alimenticios por esta causa.

Sobre la base de las consideraciones anteriores, se puede mencionar que los esfuerzos reivindicatorios de la mujer en los últimos 150 años han hecho posible que posean una mayor presencia real y activa en todos los niveles de la sociedad moderna. Especialmente en el campo laboral, las mujeres han avanzado en forma estupenda gracias a las leyes y reconocimiento de su capacidad y esfuerzo. Estos avances muchas veces se ven eclipsados por las algunas mujeres quienes con su comportamiento competitivo, defensivo o poco solidario, especialmente con sus pares en el trabajo o en cualquier ambiente como las instituciones educativas, podrían llegar a crear condiciones desfavorables las cuales afectan la productividad y armonía de una oficina, salón de clases, taller o una entidad gubernamental. En el ámbito social, esa competencia poco constructiva suele verse reflejada en acciones incomprensibles donde en vez de unirse por una causa común en muchas ocasiones podrían estar en disputa. La vida familiar es otro campo en donde las relaciones de mujeres muchas veces son devastadoras para la armonía y desarrollo del núcleo que es el eje de toda sociedad (Ludwig y Marble, 2004).

\section{Resultados}

Zizek (2008), señala dos tipos esenciales de violencia: la violencia subjetiva y la objetiva. Para él, la violencia subjetiva es aquella que se manifiesta de forma individual, en la cual el agresor expresa una posición particular frente a la víctima. En este tipo de violencia se recogen las modalidades reconocibles como el robo, los asesinatos a sangre fría, las violaciones, etc. La violencia subjetiva genera desorden, caos, crisis y exige el retorno del orden. La violencia subjetiva, aunque es la más espectacular (dada la cobertura que usualmente le hacen los medios de comunicación), no es la más devastadora, si se evalúan los efectos que ocasiona. En referencia a la clasificación anterior, es importante destacar las dos formas de violencia objetiva, complementarias con la violencia subjetiva. Estas son: la violencia simbólica y la violencia sistémica, las cuales definiremos a continuación:

- Violencia simbólica: toda manifestación discursiva que, de una u otra forma, agrede al otro por su condición de diversidad, ejemplos: el lenguaje de odio interracial, de clase, sexual, así como las múltiples formas de discriminación -incluidas las prácticas de apoyo y solidaridad para con los "menos favorecidos" -, pues estas logran perpetuar el status quo de 
una sociedad escindida, que se niega a generar transformaciones radicales en función de las diferencias de etnia y clase.

- Violencia sistémica: ejercida ya no por personas sino por ideologías políticas y relatos sociales sostenidos por los actuales regímenes económicos y políticos a escala mundial: el capitalismo. Sus prácticas de libre mercado y estimulación a la competencia económica; las nuevas modalidades de racismo, amparadas en la biología y la higiene social; y la biopolítica contemporánea, etc. Para Zizek (2008), los factores mencionados, son los causantes de la perpetuación de las diferencias de clase, de conflictos inter étnicos y del establecimiento de modos de vida que se convierten en dominantes (hegemónicos). Es en este punto de quiebre conceptual en el que se señala la diferencia entre las dos expresiones de violencia: la violencia objetiva opera, no como un agente desestabilizador de la sociedad que convoca al caos y al desorden, sino como un mecanismo de perpetuación del orden actual. La violencia objetiva, tal como la concibe Zizek (2008), no es crisis si no perpetuación del orden "normal" de las cosas.

\section{Aspectos demográficos de las alumnas}

En este apartado se analiza las características demográficas de las adolescentes de la Universidad Autónoma de Nuevo León y la preparatoria técnica CONALEP. Se puede apreciar en la figura 1 que la mayoría de las alumnas a quienes se aplicó el cuestionario tienen entre 16 y 17 años. Debemos explicar que es la edad en la que normalmente las alumnas se encuentran un poco más estables en la preparatoria ya que típicamente ingresan a los 15 años, observándose después una especie de filtro en el cual para el siguiente año están quienes tienen más probabilidades de terminar sus estudios de Nivel Medio Superior y quienes también de alguna manera se han habituado a su entorno escolar y esto probablemente las lleve a desenvolverse con más confianza mostrando un comportamiento real. Por otra parte, las alumnas de 18 y 19 años no se encuentran en la edad promedio de cursar la preparatoria técnica ya que entraron tarde al Nivel Medio Superior por algún motivo especial. Por otra parte, es importante señalar que 11 alumnas no respondieron sobre su edad actual (figura 1).

Así mismo, cuando analizamos el lugar de residencia de las adolescentes en la Zona Metropolitana de Monterrey, podemos observar que la mayoría de las estudiantes viven en los municipios de Apodaca y Guadalupe siendo que las preparatorias en donde estudian están

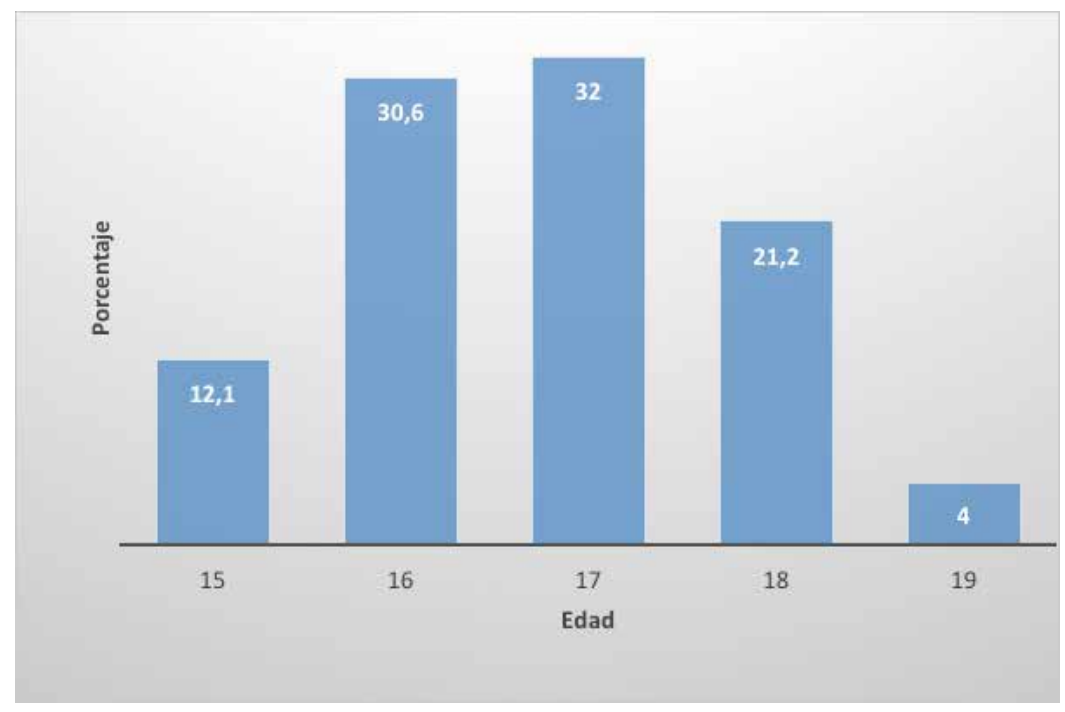

Figura 1. Edad cumplida de las adolescentes en las preparatorias técnicas 
ubicadas en Monterrey, a pesar de que en sus propios municipios existen ofertas educativas de bachillerato técnico como el CONALEP 289 "Dr. Carlos Canseco González" y el Centro de Bachillerato Tecnológico Industrial y de Servicios Núm. 53 ubicados en Apodaca, N.L., y la Escuela Industrial y Preparatoria Técnica Álvaro Obregón Unidad Guadalupe y el CONALEP "Ing. Raúl Rangel Frías" ubicados en el municipio de Guadalupe (ver figura 2).

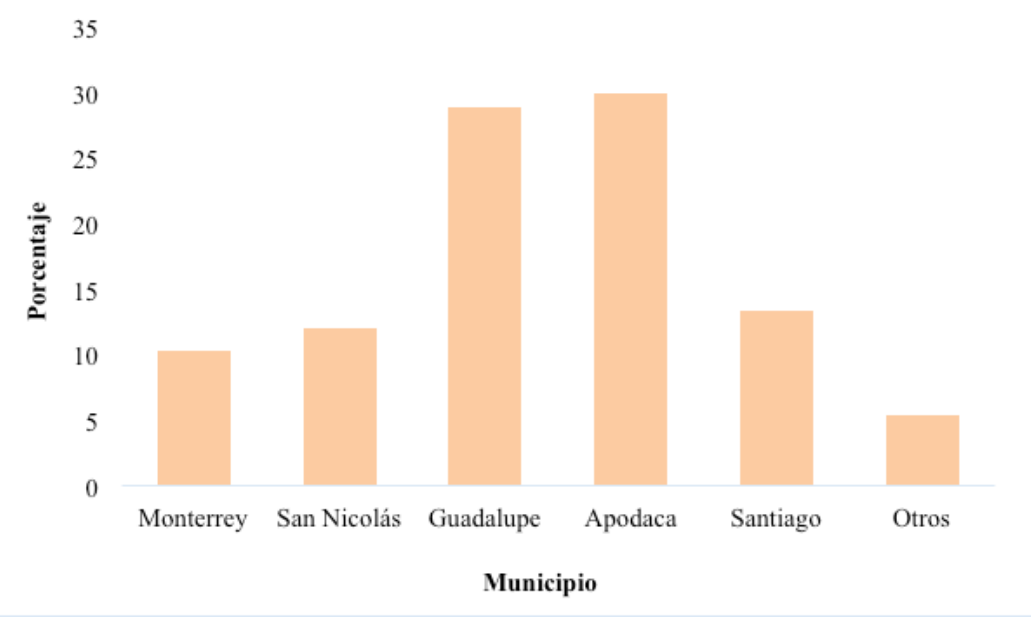

Figura 2. Municipio en el que viven las adolescentes de las preparatorias técnicas

Por otra parte cuando analizamos las especialidades que están cursando las alumnas de preparatoria, se puede apreciar son 275 alumnas respondieron la carrera que cursan y la mayor población pertenece a las carreras de artes gráficas (45.8 por ciento), trabajo social (32.8 por ciento) y tecnología de la información y computación (10.5 por ciento). Esto se debe a que se ha observado que típicamente son las especialidades con mayor población femenina. Sin embargo, también nuestro análisis señala que hay alumnas que están estudiando máquinas y herramientas, trabajo social, electromecánica, mecatrónica, estadística y aeronáutica (ver figura 3).

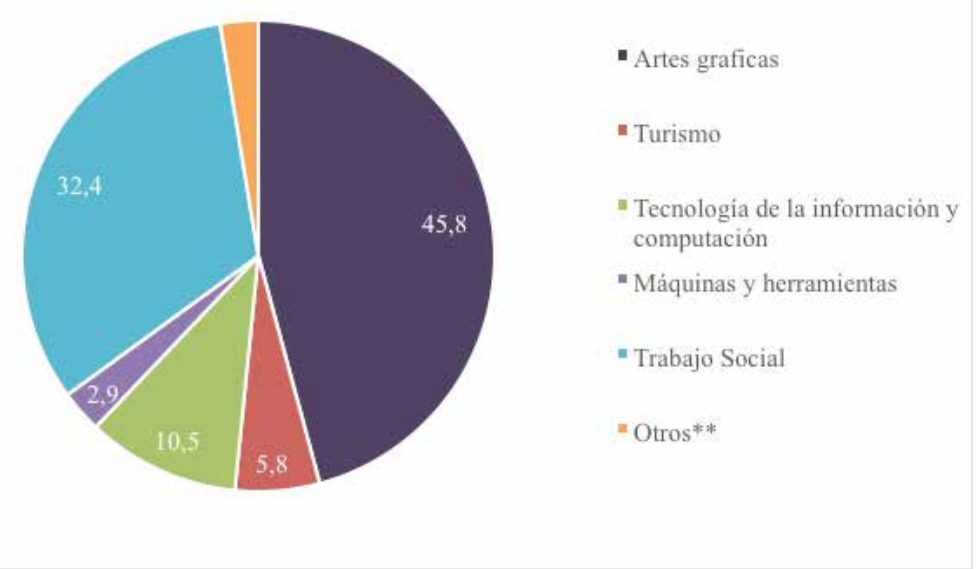

Figura 3. Programa que estudian las adolescentes en las preparatorias técnicas 
En este caso es necesario observar que las alumnas durante la preparatoria experimentan una transición en la cual algunos aspectos toman mayor relevancia que antes, como el estatus social de su familia, qué marca de teléfono celular posee, la ropa que viste, su apariencia física e incluso la disputa por algún novio podrían ser motivo de un comportamiento violento, además los gustos, la personalidad y en general la idea de la orientación que desean dar a su vida podrían estar un poco más claras que cuando recién van entrando de la secundaria a la preparatoria, en ese sentido, podría favorecernos a nuestro estudio que el 64 por ciento de las alumnas en cuestión cursan del 4-8 semestre y el 36 por ciento cursan del 1-3 semestre (ver tabla 1).

De igual manera cabe mencionar que el turno preferido de las estudiantes y sus familias para que asistan a clases es el matutino (52 por ciento) y después el vespertino (48 por ciento) debido a que se piensa que existe mayor seguridad al andar en la calle o utilizar el transporte público con luz de día, las alumnas tienen ya sea la mañana o la tarde libre, según sea el caso, para actividades extra escolares o bien para pasar tiempo o ayudar a la familia e incluso trabajar un medio tiempo si así lo desean.

Tabla 1: Semestre y turno que estudian las adolescentes en las preparatorias técnicas

\begin{tabular}{|l|l|l|}
\hline & Porcentaje & Número \\
\hline Semestre que cursa (n=289) & & \\
1 a 3 & 65.98 & 104 \\
4 a 8 & 64.01 & 185 \\
Turno que cursa (n=291) & & \\
Matutino & 51.9 & 151 \\
Vespertino & 47.8 & 139 \\
Nocturno & 0.3 & 1 \\
\hline
\end{tabular}

Observación de violencia en la familia: Violencia objetiva-simbólica

Según Urie Bronfrenbrenner (1979 en Latorre, 2011) en la Teoría Ecológica sobre el desarrollo y el cambio de conducta; el ambiente influye en el sujeto y en el cambio que experimenta durante su desarrollo, con lo cual, supone una de las teorías más emergentes y aceptadas de la psicología evolutiva actual. Los ambientes de la Teoría ecológica son:

\begin{tabular}{|l|l|l|l|}
\hline Microsistema & Meso sistema & Exo sistema & Macro sistema \\
\hline $\begin{array}{l}\text { Que incluye el } \\
\text { entorno familiar } \\
\text { y la escuela }\end{array}$ & $\begin{array}{l}\text { Constituido por la } \\
\text { relación familia- } \\
\text { escuela-amigos }\end{array}$ & $\begin{array}{l}\text { Conformado por la fami- } \\
\text { lia extensa, la influencia } \\
\text { que produce el ambiente }\end{array}$ & $\begin{array}{l}\text { Conformado por la cultura, va- } \\
\text { lores y tradiciones, el sistema } \\
\text { económico, la legislación y las } \\
\text { en el hogar, el barrio o } \\
\text { normas sociales } \\
\text { comunidad y los medios } \\
\text { de comunicación como la } \\
\text { televisión o el internet }\end{array}$ \\
\hline
\end{tabular}

Con lo anterior, es interesante observar la violencia que se da precisamente en los sistemas descritos anteriormente. Según Zizek (2008), la violencia objetiva simbólica es toda manifestación discursiva que, de una u otra forma, agrede al otro por su condición de diversidad, por ejemplo, el lenguaje de odio interracial, de clase, sexual, así como las múltiples formas de discriminación, pues éstas logran perpetuar el status quo de una sociedad escindida, que se niega a generar transformaciones radicales en función de las diferencias de etnia 
y clase. Por lo anterior hemos analizado la violencia que perciben o sufren las alumnas por parte de su familia. Para dar respuesta a esta parte, hemos realizado a las estudiantes alrededor de 16 preguntas sobre la violencia en la familia.

Dentro de esta perspectiva, la Organización Mundial de la Salud (2015) ha emitido su Estrategia Mundial para la salud de la mujer, el niño y el adolescente 2016-2030, la cual se encuentra alineada con los Objetivos de Desarrollo Sustentable (ODS) definiendo como uno de sus objetivos principales la transformación a través de la ampliación de los entornos propicios y precisamente una de las acciones propuestas para dar cumplimiento a lo anterior es eliminar todas las prácticas nocivas, la discriminación y la violencia contra las mujeres y las niñas. Debe señalarse que es la primera vez que los adolescentes ocupan, junto con las mujeres y los niños, el centro de atención de la Estrategia Mundial reconociendo no solamente los problemas sanitarios específicos que afectan a los jóvenes, sino también la función fundamental que han de desempeñar, junto con las mujeres y los niños, como impulsores clave del cambio en la era posterior a 2015. Dicha estrategia defiende la idea de que al invertir en las políticas y los programas adecuados para realizar el potencial de los adolescentes y sus derechos humanos a la salud, la educación y la plena participación en la sociedad, podría liberarse el enorme potencial humano de esta «generación de los ODS» para transformar nuestro mundo.

En relación a la problemática expuesta, el 26.2 por ciento de las adolescentes dijeron que sus padres o tutores las controlan constantemente (ver figura 4) por diferentes razones, entre las cuales se encuentran que sus familiares son autoritarios y a la vez sobre protectores siendo lo anterior un dato curioso, ya que se pueden observar opiniones encontradas debido a que algunas adolescentes piensan que el grado de control de los padres se debe a que solamente desean lo mejor para ellas, como por ejemplo evitar que malgasten el dinero, que vistan con mal gusto o simplemente porque fueron los padres quienes les compraron lo que tienen; a este respecto algunas alumnas dijeron sentir decepción e impotencia de que los padres quieren decirles qué hacer con el dinero que ganan en sus respectivos trabajos, e incluso algunas estudiantes admitieron sentirse humilladas ya que sus padres les piden sus teléfonos celulares para revisarlos viéndose con esto sumamente invadidas en su espacio y privacidad. Como un ejemplo de lo anterior, una estudiante de 16 años de la Preparatoria 20 de la UANL comentó: mis padres siempre me están preguntando qué tanto estoy hablando con mi celular, molestándome cada vez que llamo o me llaman mis amigas y es peor cuando quiero hablar con mis amigos porque no quieren que tenga novio, y cada

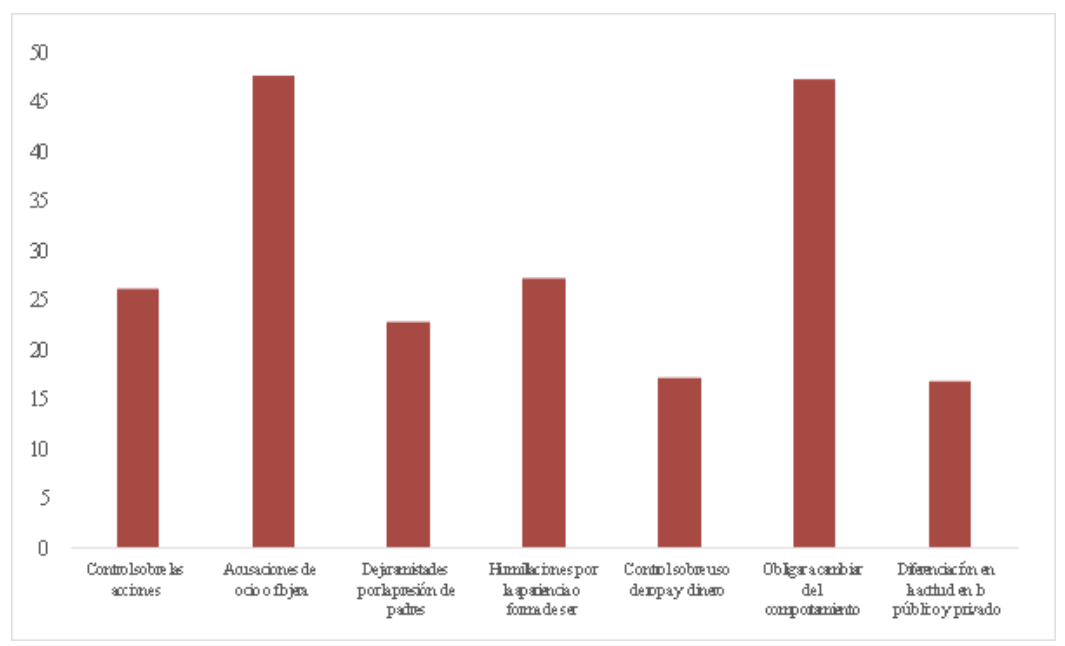

Figura 4. Violencia objetiva-simbólica que sufren las adolescentes en familia 
cierto tiempo les tengo que entregar mi celular a mis padres para que lo revisen y no puedo oponerme porque me gritan que son ellos quienes me compraron el celular y quienes pagan la factura de las llamadas y aunque así sea, me hacen sentir humillada e invadida en mi privacidad y que ya no puedo estar en paz ni un momento porque siempre hay alguien vigilándome.

Por otra parte, el 47.5 por ciento de las adolescentes se sienten violentadas al ser acusadas constantemente de ocio o flojera por parte de sus padres o tutores percibiendo que las principales causas de esto son que no colaboran con los quehaceres del hogar y que pasan mucho tiempo acostadas, en relación a esto, algunas alumnas comentaron que sus padres se desesperan porque ellas no buscan trabajo mostrándose éstos malhumorados porque ellas no se apuraban a buscar trabajo haciéndolas sentir como una carga para la familia. En este sentido una alumna de 17 años de la escuela CONALEP, comentó: cuando llego del entrenamiento me siento muy cansada y voy a acostarme un rato y en cuanto mis padres me ven descansando me dicen que soy una floja y que no ayudo en nada en la casa, en otras ocasiones ni siquiera puedo ver televisión tranquila porque ya me están diciendo que si no tengo que hacer me ponga a limpiar o a hacer cualquier otra cosa, que de perdido me gane lo que me como, lo cual me hace sentir bastante mal porque siento que no puedo tener ni un minuto de descanso sin que mis padres me estén molestando.

Además, el 22.8 por ciento de las estudiantes dijeron haber perdido contacto con familiares, amigas(os) y/o compañeras(os) para evitar que sus padres o tutores se molesten, ya que sus familiares argumentan que dichas compañías no les agradan debido a que son malas personas y ellas dejan sus amistades tienden a evitar los regaños principalmente; sin embargo el recelo de ciertos padres podría deberse también a otras razones ocultas ya que por ejemplo, al menos un par de alumnas manifestaron detectar "conductas extrañas" en sus padres como espiarlas o mirarlas de manera incómoda y a veces tratarlas con exceso de cariño, o bien éstos mostrarse celosos de que ellas entablen amistad con adolescentes del sexo masculino por la posibilidad de que pudieran hacerse novios.

En relación con lo anterior, la psicóloga y pedagoga San Sebastián (2008) comenta que en la niñez los padres suelen ejercer un grado elevado de control sobre sus hijos, sin embargo esta postura no debería perpetuarse hasta la adolescencia ya que la percepción de los hijos en esta etapa cambia, viendo a los padres más humanos y más susceptibles a cometer errores a diferencia a cómo los veían en su niñez como los más listos o los mejores. Por lo anterior, la psicóloga comenta que los padres deberían escuchar más y hablar menos, estar presentes para los hijos adolescentes marcando cierta distancia pero sin desaparecer, buscando con ello que sea el adolescente quien marque la pauta acerca de en qué momento solicitar el apoyo y orientación de los padres y hablar.

Se observa también que otra forma en la cual las estudiantes perciben ser violentadas es lo que conceptualizan como humillación por parte de los padres o tutores, entendido como un sentimiento de vergüenza que experimentan cuando son criticadas ya sea por la apariencia o forma de ser, y estas críticas son realizadas en público o privado.

En relación a lo anterior, la psicóloga clínica y de salud De Francisco (2015) explica que los adolescentes empiezan a vivir experiencias con más valor y emocionalidad existiendo a su vez contradicciones debido a que, por una parte, mantienen conductas infantiles pero, por otra, dan indicios de independencia. Se hace manifiesta la autocrítica y además la crítica al mundo que les rodea, buscando su espacio e intimidad, su autonomía y la diferenciación de su mundo con respecto al de sus padres. Según la Organización Mundial de la Salud (2015), la adolescencia es una de las etapas de transición más importantes en la vida del ser humano, que se caracteriza por un ritmo acelerado de crecimiento y de cambios, superado únicamente por el que experimentan los lactantes. Tal es caso del 27.2 por ciento de las adolescentes cuestionadas, quienes mencionaron que sus padres o tutores las critican y/o humillan por cuestiones como su apariencia, su forma de ser, el modo en el que hacen sus tareas, etc., 
señalando como principales causas de esto que los gustos de sus familiares son diferentes a los de ellas y que así son los padres por naturaleza.

Así mismo, se observa que el 26.4 por ciento de las adolescentes mencionó que sus padres las critican tanto en público como en privado, y de ese porcentaje el 41.3 por ciento tiene una percepción positiva ya que mencionaron que las critican en privado de manera constructiva para mejorar y el 17.5 por ciento también en privado por tratarse de cuestiones familiares, sin embargo el porcentaje restante siente haber sido humillada en público como una forma de molestarla, para hacerla sentir mal, por ignorancia de los padres o tutores o bien simplemente para modificar su comportamiento. Así mismo, algunas alumnas comentaron haber sido humilladas y avergonzadas cuando sus padrastros actuaron de manera distinta de lo habitual estando frente a sus madres o frente a la familia de ésta, ya sea regañándolas o dándoles consejos, a los ojos de las alumnas exagerados o innecesarios, con el único objetivo de lucirse y quedar bien haciéndose pasar como el padre súper héroe.

\section{Observación de violencia en la familia: Violencia subjetiva}

Retomando a Slavoj Zizek (2008), mencionaremos su concepto de la violencia subjetiva que es aquella que se manifiesta de forma individual, en la cual el agresor expresa una posición particular frente a la víctima. Este tipo de violencia es la más aparatosa y evidente, ya que tiene efectos más reconocibles generando de inmediato caos y crisis, en ese sentido la violencia física podría ser encasillada dentro de esta modalidad. A este respecto en la investigación se descubrió que el 65 por ciento de las alumnas han sido golpeadas con algún objeto o con la mano y el 7 por ciento manifiesta haber sido amenazada o bien, haber presenciado amenazas hacia otro miembro de la familia con armas con la intención de matar (ver tabla 2). Por ejemplo, podemos citar que algunas alumnas declararon haber sido testigos por lo menos una vez de que el padre o la madre apretara el cuello de su pareja con la intención de ahorcarlo, incluso una alumna comentó haber sido ella misma tomada por el cuello por su padre porque éste consideró que le había contestado de manera bastante grosera. En este sentido una alumna de 17 años de la escuela CONALEP dijo: hubo una vez en la que mi padre llegó ebrio y tarde a casa, y entonces mi mamá se puso histérica y empezó a gritarle ahorcándolo y mi mamá al escuchar que mis hermanos y yo comenzamos a gritar y llorar lo soltó.

Dado lo anterior, no existe un perfil específico de los padres maltratadores, pero si se sabe que pertenecen a todas las clases sociales, su nivel educativo es muy diverso y muy pocos padecen alguna especie de patología mental, sin embargo, investigaciones recientes nos permiten hablar de factores de riesgo que propician que los padres tengan conductas violentas con sus hijos como por ejemplo la creencia de que el castigo corporal es por su bien, o también porque los padres argumentan que a ellos los educaron así. En relación a lo anterior algunas alumnas dijeron haber recibido palizas por parte de sus padres por diversas razones que van desde contestar mal o llegar muy tarde a casa hasta ser sorprendidas teniendo relaciones sexuales, admitiendo haber aceptado la golpiza con resignación ya que consideraron merecerla o que sus padres lo hicieron por su propio bien, sin embargo otras comentaron que habían tratado de defenderse solamente llorando o gritando. Un caso relacionado con lo anterior lo comentó una estudiante de 16 años del CONALEP: una tarde mi novio y yo nos fuimos a mi casa saliendo de clases para hacer la tarea y como no estaban mis papás nos fuimos a mi cuarto y se nos hizo fácil aprovechar para tener relaciones, pero yo no me había dado cuenta que mi papá estaba dormido en su cuarto y cuando escuchó ruidos fue a revisar y nos encontró en plena acción; en ese instante mi papá le aventó patadas a mi novio y lo persiguió hasta que se salió de la casa y luego se regresó conmigo y me pegó y me agarró por el cuello. Yo no hice nada porque en ese momento sentí mucho miedo y vergüenza, solamente lloré mucho hasta que mi papá me soltó. 
Por otra parte, existen muchos padres que poseen un convencimiento de que los hijos les pertenecen, derivado ya sea de tradiciones o creencias religiosas. Algunos otros padres experimentan una incapacidad de controlar sus emociones, manifestando expresiones inadecuadas de rabia y una baja tolerancia a la frustración. A este respecto, por lo menos un par de estudiantes mencionaron que alguno de los padres recurriría a medicamentos psiquiátricos como ansiolíticos o anti-depresivos, dependiendo de éstos para tener un comportamiento más o menos estable y que el día que las píldoras se terminaban o no había dinero para comprarlas debían pasar por una situación bastante angustiante ya que esto se traducía en un periodo de maltrato hacia ellas y los demás integrantes de la familia, el cual se prolongaba hasta que la situación económica volviera a la normalidad.

Además, hay padres que incluso dotan de una intencionalidad negativa al comportamiento de sus hijos, acusándolos de que actúan así para molestarlos y por último, los padres también han argumentado que no tienen quien les ayude evidenciando de esta manera a una red deficiente de apoyo social (Castillo, 2006). Al respecto, en la tabla 2 podemos observar que un 65.1 por ciento de las alumnas dijeron haber sido golpeadas con algún objeto o con la mano o bien haber sido lastimadas físicamente por los padres o tutores y el 7 por ciento comentaron haber recibido amenazas con objetos o armas con la intención de matar. Como causas de lo anterior la mayoría de las alumnas admitieron haberse portado mal, es decir haberse "pasado" con algún mal comportamiento, y como mal comportamiento refirieron desde haber llegado muy tarde a su casa o contestar feo a sus padres hasta llegar alcoholizadas o sostener relaciones sexuales con adultos. Otros factores que citaron como causas de ser golpeadas fueron que los padres suelen tener un carácter muy fuerte o bien estar enojados constantemente.

Tabla 2: Violencia subjetiva que sufren las adolescentes por parte de sus padres o tutores

\begin{tabular}{|l|l|l|}
\hline Violencia subjetiva & Porcentaje & Numero \\
\hline $\begin{array}{l}\text { Golpeado con objeto o mano o lastimado físicamente } \\
\text { Amenazas con objeto o armas para matar }\end{array}$ & 65.1 & 196 \\
\hline
\end{tabular}

Agresión en la escuela a consecuencia de la violencia en el entorno familiar

Aunque es en la escuela, el espacio donde se dan lugar a los comportamientos agresivos, no siempre se generan ahí, sino que están relacionados con otros ámbitos, como el de la violencia en la familia, la cual se desempeña como el primer medio socializador del niño y es donde debe recibir los primeros afectos y vínculos materno y paterno, y es aquí donde también se constituye en un elemento fundamental para la génesis de las conductas agresivas de los hijos que repercutirán hasta la vida adulta al afectar su forma de integración y de relación social. En un enfoque ideal, la familia constituye el sistema de relación primario del ser humano y desde su nacimiento debe vivir la satisfacción de sus necesidades básicas más elementales, aunado a eso la familia cumple una función socializante muy significativa, es decir, es un grupo de relación que marca y determina el proceso de desarrollo humano. La familia es esencial para comprender el carácter de un niño agresivo o sus conductas conflictivas. Ante eso, es esencial destacar el papel que los padres tienen en el proceso de desarrollo de sus hijos así como su implicación en la emergencia de conductas antisociales. De ahí que los padres también se tomen en cuenta en los estudios sobre violencia escolar, junto con los docentes y los alumnos (Pérez, et.al, 2008 en Perea, et.al., 2010).

Así mismo, existen factores que favorecen el desarrollo de la agresión en el individuo, destacándose como más relevantes los siguientes (Carbonero, et.al; 2002 en Perea et al. 2010): 
1. Factores biológicos: donde muestran una predisposición biológica hacia las conductas desadaptadas.

2. Factores ambientales: se estudia la influencia familiar porque es el ambiente en la que se inicia la conducta del sujeto. La mayoría de los estudios realizados intentan precisar las características de las relaciones familiares y el alcance de su implicación en las conductas agresivas de los individuos.

3. Factores cognitivos y sociales: en la cual los individuos no poseen respuestas ante situaciones adversas que no sean agresivas, y aplican la agresividad como forma de interactuar con el medio lo cual, constituye una inadaptación ocasionada por problemas en la codificación de la información, lo cual dificulta la capacidad de generar respuestas alternativas. Por ejemplo, una de las alumnas de la carrera de artes gráficas de 16 años a quien se le aplicó el cuestionario dentro de nuestra investigación de campo comentó: mi mamá me dice que "me la lleve tranquila", que no me pelee, pero que si me provocan que entonces si le entre a los trancazos porque si yo no me defendía, luego ella me iba a pegar a mi "por dejada". Respuestas parecidas dieron otras alumnas cuestionadas en otros semestres del turno vespertino de la misma carrera, en cuanto a los consejos que sus padres les dan acerca de responder con violencia cuando a su vez ellas son violentadas.

4. Factores de personalidad: en donde se establece las características de personalidad asociadas a las conductas agresivas del individuo, las cuales serían por ejemplo despreocupación por los demás, burlarse de los demás y ponerles en ridículo y crueldad e insensibilidad ante los problemas de los demás, por mencionar algunas. Por ejemplo, en nuestra investigación realizada en una de las preparatorias técnicas a las que acudimos, una alumna de 17 años del cuarto semestre también de la carrera de artes gráficas mencionó: yo me junto con unas amigas y "matamos el tiempo acarrillando" nos burlamos cuando se portan distinto o si vienen vestidas ridículas nomás para que los demás del grupo también se diviertan”. Cuando a esta alumna se le cuestionó de que si experimentaba alguna pena por los sentimientos de las chicas agredidas, ella contestó que no era importante, y que luego "se les pasaría".

Los factores anteriormente mencionados han dirigido la atención hacia la familia como un factor explicativo, en ellos se comprueba la influencia familiar en los individuos, tanto en un correcto ajuste psicosocial como en su implicación en conductas problemáticas como el consumo de sustancias o las conductas violentas (Dekovic, et.al; 2004; Martínez, et.al;, 2003; Musitu, et.al; 2001 en Perea et al. 2010).

Después de lo anteriormente expuesto, es importante destacar que la familia está en crisis, el tejido social se ha dañado de manera importante, las familias disfuncionales e inseguras atentan con destruir lo que en teoría debería ser un entorno familiar, sin embargo cabe señalar que la familia constituye un componente de vital importancia para las sociedades civilizadas. De hecho, podríamos asumir que en un breve periodo de tiempo se ha pasado de una tradicional familia unida, a una en la cual los integrantes son más independientes, desde temprana edad.

Sobre la base de las consideraciones anteriores, es preciso señalar algunos de los factores de riesgo los cuales impactan en la vida del estudiante violento (Fernández, 2008 en Perea et al. 2010), como son:

- Los malos tratos y el modelo violento dentro de la familia que enseña al individuo a resolver los conflictos mediante la agresión física o verbal.

- La disfunción familiar que lleva a la falta de atención hacia los hijos. 
- El modelo de familia que enseña al individuo a ejercer el poder sobre otro más débil, así como la falta de negociación y de diálogo.

- Los métodos de crianza con prácticas excesivamente laxas e inconsistentes o en caso contrario muy restrictivos y excesivamente punitivos.

- La falta de afecto entre cónyuges con ausencia de seguridad y cariño, lo que provoca conflictos entre todos los integrantes.

\section{Conclusiones}

Como se observa, la violencia familiar se ha convertido en uno de los problemas fundamentales de la salud pública. La exposición constante de los jóvenes a la violencia familiar tiene graves consecuencias en su desarrollo cognitivo, afectivo, social y de salud trayendo consecuencias que van desde dolores de cabeza y/o estómago, estrés y angustia hasta estados depresivos, además de que podrían presentar también problemas de conducta y adicciones. En relación a lo anterior, estudios han demostrado que los hijos tienden a repetir los patrones de violencia vividos en sus hogares y por otra parte es interesante mencionar que se han encontrado diferencias en la violencia intrafamiliar ejercida entre géneros, por ejemplo algunos estudios reportan una mayor agresión hacia mujeres adolescentes en comparación con los hombres siendo una posible explicación que este comportamiento se basa en una mayor preocupación de los padres sobre la autonomía de las hijas, especialmente en el aspecto de su sexualidad. Además, de que es más difícil maltratar a los varones por su mayor tamaño y fuerza. Por otra parte, es bien sabido la gran aceptación que tiene un varón naturalmente violento con el privilegio que la misma sociedad le otorga de ejercer la violencia, en contraste con la mujeres a quienes los estereotipos de género asocian como un ser sometido y victimizado.

\section{Bibliografía}

Acevedo, J. (2012). ¡Tengo Miedo! Violencia en las Escuelas: La violencia y su percepción social. México, D.F.: Trillas.

Acharya, Arun Kumar y Salas Stevanato, Adriana (2005), Violencia y tráfico de mujeres en México: una perspectiva de género, Revista Estudos Feministas, vol. 13, núm. 3, septiembrediciembre, pp. 507-524.

Acharya, A.K. (2016), Trafficking of women for sexual exploitation in Mexico and their identity crisis, International Review of Sociology, 26(2), 322-336.

Ahlers, A. (2011), Mentiras estúpidas que las mujeres se dicen a sí mismas. (pp. 13-20). México, D.F. Aguilar.

Almeida, C. \& Gómez, M. (2005), Las huellas de la violencia invisible. Barcelona, España. Ariel.

Amaya, C., Acharya, A. and Bonfiglio, J. (2016), Gender Based Violence and Reproductive Health of Indigenous Women in Mexico, Sociology Mind, 6, 107-113. doi: 10.4236/sm.2016.63009.

Conciencia Educativa (2011), Análisis: Teoría Bioecológica Bronfrenbrenner: Medio Ambiente y Aprendizaje. [En línea]. Daniela Latorre. Santiago de Chile. <http://concienciaeducativalatorred.blogspot.mx/2011/10/analisis-teoria-bioecologica.html> [Consulta: 3 feb. 2016].

El País (2015), Papá, no me avergüences. Los adolescentes se desahogan [en línea]. Belén De Francisco. América, <http://elpais.com/elpais/2015/10/28/icon/1446031051_707770.html > [Consulta: 1 mar. 2017].

Elnuevodiario.com.ni. Niñas no se sienten seguras en el Colegio. [en línea]. Leyla Jarquín. Managua, Nicaragua, 2014. < http://www.elnuevodiario.com.ni/nacionales/332806-ninas-nose-sienten-seguras-colegio/ > [Consulta: 5 abr. 2017].

Ludwig, T. \& Marble, A. (2004), Mi Enemiga Secreta: Se revela el secreto de la agresión emocional. Nueva York: River Wood Books.

Moser, C., \& Mcllwaine, C. (2005), La Violencia Urbana en Latinoamérica como problema de Desarrrollo: hacia un Marco para reducir la violencia. Santiago de Chile.

Organización Mundial de la Salud. Estrategia mundial para la salud de la mujer, el niño y el adoles- 
cente (2016-2030) [en línea]. Amina Mohammed. Ginebra, Suiza 2015. <http://www.who. int/maternal_child_adolescent/documents/women-deliver-global-strategy/es/ > [Consulta: 18 feb. 2017].

Perea, M., Calvo, A. \& Anguiano, A. (2010). La familia y la escuela coexistiendo con la violencia escolar, Margen, 58. Recuperado desde: https://www.margen.org/suscri/margen58/perea. pdf

Polegar Medios. Excesivo control sobre los hijos adolescentes, suele provocar la reacción contraria [en línea]. Maribel San Sebastián. España, 2017. <http://guiajuvenil.com/comunicacion/ excesivo-control-sobre-los-hijos-adolescentes-suele-provocar-la-reaccion-cont.html > [Consulta: 21 mar. 2017].

Psicopedagogia.com. Padres que maltratan a sus hijos [en línea]. Neyla Castillo. Madrid, 2006. $<$ http://www.psicopedagogia.com/padres-maltratan > [Consulta: 23 mar. 2017].

Psychosocial Intervention. Violencia escolar: Un análisis desde los diferentes contextos de interacción [en línea]. Varela, R., Ávila, M., Martínez, B. Madrid, 2013). http://psychosocial-intervention.elsevier.es/es/violencia-escolar-un-analisis-desde/articulo/S1132055913700048/\#. WSVVy5I1_IV [Consulta: 15 ago. 2015].

Secretaría de Educación Pública (2011). Manual de Seguridad Escolar. Sindicato Nacional de Trabajadores de la Educación. México.

Suárez, L., \& Menkes, C. (2006). Violencia Familiar ejercida en contra de los adolescentes mexicanos. Cuernavaca, Morelos, México.

Viguer, P., \& Avià, S. (2009). Un modelo local para la promoción de la convivencia y la prevención de la violencia entre iguales desde el ámbito comunitario. Valencia, España.

Zikek, S. (2008). Sobre la violencia. Seis Reflexiones Marginales. Barcelona: Paidós Ibérica. 